\title{
Analyzing the impact of the number of nodes on the performance of the routing protocols in manet environment
}

\author{
Hussein M. Haglan ${ }^{1}$, Salama A. Mostafa ${ }^{2}$, Noor Zuraidin Mohd Safar ${ }^{3}$, Aida Mustapha ${ }^{4}$, \\ Mohd. Zainuri Saringatb ${ }^{5}$, Hosam Alhakami ${ }^{6}$, Wajdi Alhakami \\ ${ }^{1}$ Computer Center, University of Anbar, Iraq \\ 2,3,4,5 Faculty of Computer Science and Information Technology, Universiti Tun Hussin Onn Malaysia, Malaysia \\ ${ }^{6}$ Department of Computer Science, College of Computer \& Information Systems, Umm Al-Qura University, Saudi Arabia \\ ${ }^{7}$ College of Computer Science and Technology, Taif University, Saudi Arabia
}

\begin{tabular}{|c|c|}
\hline Article Info & ABSTRACT \\
\hline Article history: & \multirow{12}{*}{$\begin{array}{l}\text { Mobile Ad-hoc Networks (MANETs) are independent systems that can work } \\
\text { without the requirement for unified controls, pre-setup to the paths/routes or } \\
\text { advance communication structures. The nodes/hubs of a MANET are } \\
\text { independently controlled, which permit them to behave unreservedly in a } \\
\text { randomized way inside the MANET. The hubs can leave their MANET and } \\
\text { join different MANETs whenever the need arises. These attributes, in any } \\
\text { case, may contrarily influence the performance of the routing conventions (or } \\
\text { protocols) and the general topology of the systems. Along these lines, } \\
\text { MANETs include uniquely planned routing conventions that responsively as } \\
\text { well as proactively carry out the routing. This paper assesses and looks at the } \\
\text { effectiveness (or performance) of five directing conventions which are } \\
\text { AOMDV, DSDV, AODV, DSR and OLSR in a MANET domain. The } \\
\text { research incorporates executing a simulating environment to look at the } \\
\text { operation of the routing conventions dependent on the variable number of } \\
\text { hubs. Three evaluation indices are utilized: Throughput (TH), Packet Delivery Ratio } \\
\text { (PDR), and End-to-End delay (E2E). The assessment outcomes indicate that the } \\
\text { AODV beats other conventions in the majority of the simulated scenarios. }\end{array}$} \\
\hline Received Apr 9, 2020 & \\
\hline Revised Jun 6, 2020 & \\
\hline Accepted Aug 1, 2020 & \\
\hline Keywords: & \\
\hline AODV & \\
\hline AOMDV & \\
\hline DSDV & \\
\hline DSR & \\
\hline MANET & \\
\hline OLSR & \\
\hline & \\
\hline
\end{tabular}

This is an open access article under the $\underline{C C B Y-S A}$ license.

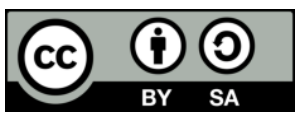

\section{Corresponding Author:}

Salama A. Mostafa,

Faculty of Computer Science and Information Technology,

Universiti Tun Hussin Onn Malaysia,

86400, Parit Raja, Batu Pahat, Johor, Malaysia.

Email: salama@uthm.edu.my

\section{INTRODUCTION}

A Mobile Ad-hoc Network (MANET) comprises of a gathering of remote hubs that can be dynamically set up to work freely from the framework prerequisite [1,2]. The system hubs have an independent design in which the hubs get signals from cell phones or portable mobile gadgets that are associated using remote/wireless connections. The hubs move haphazardly inside the system in the absence of consistent requirements or standards. These hubs go about as routing gadgets that work inside a similar topology as of the encompassing systems and building up unique associations, links or connections [3-5]. Moreover, if there is no pre-introduced base station, the hubs would not have the option to uninhibitedly or freely move inside systems. Coordinated towards a multi-hop (multiple bounces) frameworks are data packets due to the restricted radio transfer speed (or bandwidth) in the broadcast of every hub. There are a few related issues with the MANET structure. The MANET utilizes a multi-hop routing convention because 
of the limitation of the system with a scope of remote/wireless radios to shield against intrusions [6, 7]. The convention may ruin the determination of a fitting or suitable routing that is more effective than others. A few routing conventions with various direction procedures are recommended, for example, movement limitation, increment in power usage, decline the available data transfer capacity, and high accuracy rates despite the piece $[8,9]$. The primary contrasts between these methodologies are the component used to refresh data/information direction.

In recent times, the progression in the remote system study and implementations is by all accounts decentralization, consequently MANET development. The MANET is typified by a framework-less system in which every one of its remote gadgets is considered as a self-ruling element and can be known as multihop remote frameworks $[10,11]$. There are two primary roles of MANET hubs in the system which are facilitating (hosting) and routing [12-14]. With these capacities, MANET hubs straightforwardly or by implication converse with one another over a radio transmission range. The irregular (or random) versatility attribute of the system and radio transmission range between the hubs significantly alter the system topology, time plan as well as areas of the remote gadgets [15-17]. A basic case of the MANET topology is as in Figure 1.

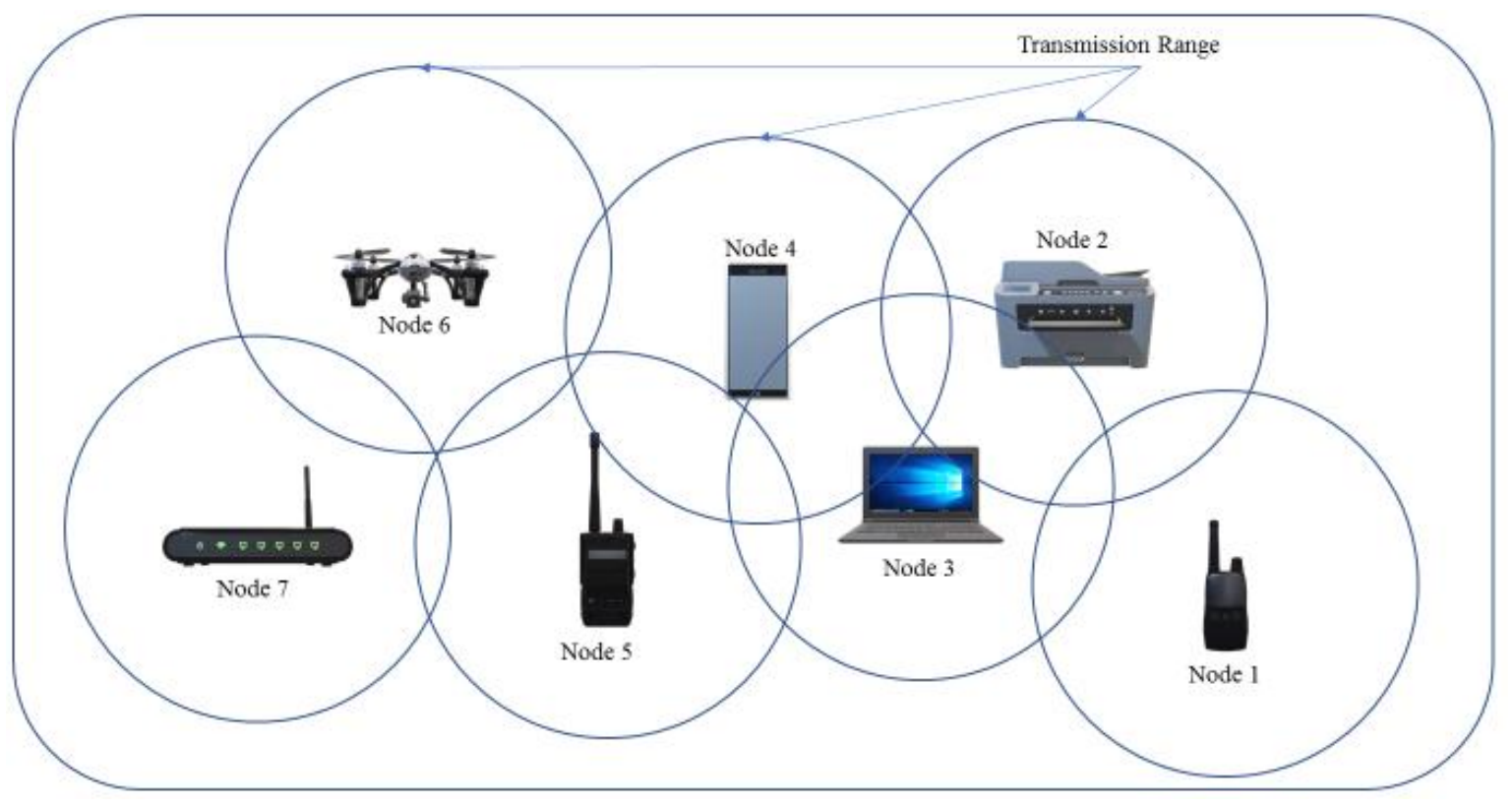

Figure 1. The MANET topology

There are many earlier research studies that have been assessed the operation of different kinds of routing conventions in MANETs, these comparatives are done with change different parameters such as density, mobility, network size and so on. Performance metrics that usually utilized to assess the performance of routing conventions are high throughput, high packet conveyance ration (or fraction), low-end-to-end delay, and low overhead and so on. In Saini and Nath [18], work on the performance of the two most generally utilized conventions DSR and AODV are assessed by altering speed and constant pause time. The investigation outcomes have demonstrated on one hand that AODV betters DSR when the speed of the hub is low and pause time remains unchanged. While AODV has performs well under the high versatility of the hubs, it was additionally discovered that the DSR has better outcomes when contrasted with AODV as far as throughput and end-to-end delay are concerned provided that interruption time remains steady. AODV, on the other hand, performs best when delay time changes.

Kumar et al. [19] looked at the performance of three conventions which are AODV, DSR, and OLSR with variation in the number and speed of nodes. The results have shown the performance of OLSR was better than DSR and AODV in average end-to-end delay and throughput with an increase in the number of nodes/hubs and their mobility. Reddy et al. [20] performance of two reactive mechanisms and three proactive mechanisms have compared by simulate AOVD and DSR as reactive protocols and DSDV, DYMO, and TORA as proactive protocols. The results have shown that topology of reactive conventions or protocols superior on the topology of proactive protocols in e2e delay and throughput. Dinesh et al. [21] compare the performance of two reactive routing protocols which are DSR and AODV with varying number 
of nodes. The results show that AODV better than DSR in TP and PDR. Kaur et al. [22] have reviewed three routing protocols which are AODV, DSDV, and DSR and compared their performances by using Network Simulator (NS2) with respect to simulation time. The results have shown that the performance of AODV is better than DSDV and DSR in terms of PDR and PLR. Although DSDV got on better TP than the other two conventions when packets generation are lesser but AODV gave better throughput than other two routing protocols when packets generation is higher.

Mai et al. [23] using DSDV, OLSR, and AODV as routing protocols in MANETs have simulated and evaluated in three scenarios which are 10, 30, 50 respectively by using NS3 as a simulation tool. Packet size was a varied parameter in each scenario. The performance indices that have been used are Throughput, Goodput, Packet Delivery Ratio, average E2E delay, and Energy Consumption (EC). The results have shown AODV as reactive routing protocol is better than other protocols, while OLSR little better other routing protocols in term of PDR. Chandra et al. [24] they have evaluated the AODV, DSR, and DSDV with a variable number of nodes. Performance metrics that have used in this evaluation are PLR, TP, E2E Delay, and Control Overhead. The results have shown AODV is better than the other two routing protocols. Aujla and Kang [25] comparative and evaluation of AODV, DSR, GRP, OLSR, and TORA routing protocols have done in two environments of applications which are Email application and video conferencing with variation density of nodes in each environment. The results have shown in video conferencing application AODV is the best in the low number of nodes but OLSR is the best in the high number of nodes. OLSR and GRP performances are the best in the Email application. GRP performance is best with the low number of nodes but regrades when the increasing number of nodes but OLSR vice versa. TORA and DSR have shown the worst performance in both scenarios. They have concluded, in both scenarios with the low number of nodes AODV and GRP are the best but with the high number of nodes, OLSR suits better as shown in Table 1.

Table 1. Summary of related work

\begin{tabular}{|c|c|c|c|c|c|c|c|c|c|}
\hline \multirow{2}{*}{ Author } & \multirow{2}{*}{ Year } & \multicolumn{6}{|c|}{ Routing Protocols } & \multirow{2}{*}{ Scenario } & \multirow{2}{*}{$\begin{array}{l}\text { Performance } \\
\text { metrics }\end{array}$} \\
\hline & & AODV & DSR & DSDV & OLSR & TORA & WRP & & \\
\hline \multirow[t]{2}{*}{ Saini and Nath } & 2018 & Yes & Yes & - & - & - & - & 1. Node speed & 1. TP \\
\hline & & & & & & & & 2. Pause time & 2. E2E delay \\
\hline Kumar et al. & & & & & & & & 2. No. of nodes & 2. E2E delay \\
\hline \multirow{2}{*}{ Reddy et al. } & 2018 & Yes & Yes & Yes & - & Yes & Yes & 1. No. of Nodes & 1. TP \\
\hline & & & & & & & & 2. Node speed & 2. E2E delay \\
\hline \multirow[t]{3}{*}{ Kaur et al. } & 2017 & Yes & Yes & Yes & - & - & - & 1.Simulation Time & 1. TP \\
\hline & & & & & & & & 2. No. of Packets & 2. PDR \\
\hline & & & & & & & & & 3. PLR \\
\hline \multirow[t]{2}{*}{ Mai et al. } & 2017 & Yes & Yes & Yes & Yes & - & - & 1. No. of Nodes & 1. TP \\
\hline & & & & & & & & 2. Packet size & 2. PDR \\
\hline \multirow{2}{*}{ Chandra et al. } & & & & & & & & & 3. E2E Delay, \\
\hline & & & & & & & & & 4. ROR \\
\hline
\end{tabular}

Table1 shows the AODV and DSR routing conventions are popularly used in different applications of MANET such, as an Email application and video conferencing. The number of nodes is a common scenario that utilized to assess the quality of these protocols. Where it has a negative effect on the network lifespan because it requires more energy to keep the active path between source and destination nodes. The TP, PDR and E2E delay is the most common metrics that use with the evaluation process. Consequently, this paper assesses the performance of five routing protocols, namely; AOMDV, DSDV, AODV, DSR and OLSR based on the number of nodes scenario under three performance indices: Throughput (TP), Packet Delivery Ratio (PDR) and End to End delay (E2E delay).

\section{MATERIALS AND METHODS}

The environment of the simulation for routing protocols are discussed in this section. The aim of this investigation is to evaluate the performance of the protocols under specific situation such, number of nodes. The main steps of the simulation model are scattered into five Modules. MANET environment built-in 
Module A. This module demonstrates the characteristics of MANET, such as a number of nodes, network size, simulation time, node speed, and so on. Second, Module B utilized to implement the five routing protocols. Third, the number of nodes scenario with several parameters was included in Module C, this module employs to evaluate the performance of the five routing protocols. Forth, Module D, the performance metrics of throughput (TP), packet delivery ratio (PDR), and end-to-end delay (E2E delay), were used to assess the performance of the protocols. Finally, a results visualization module is included to view the results and related analysis. Figure 2 is depicting the overall steps of the simulation. The simulation's parameters are implemented from [26]. Table 2 illustrates the most important simulation metrics.

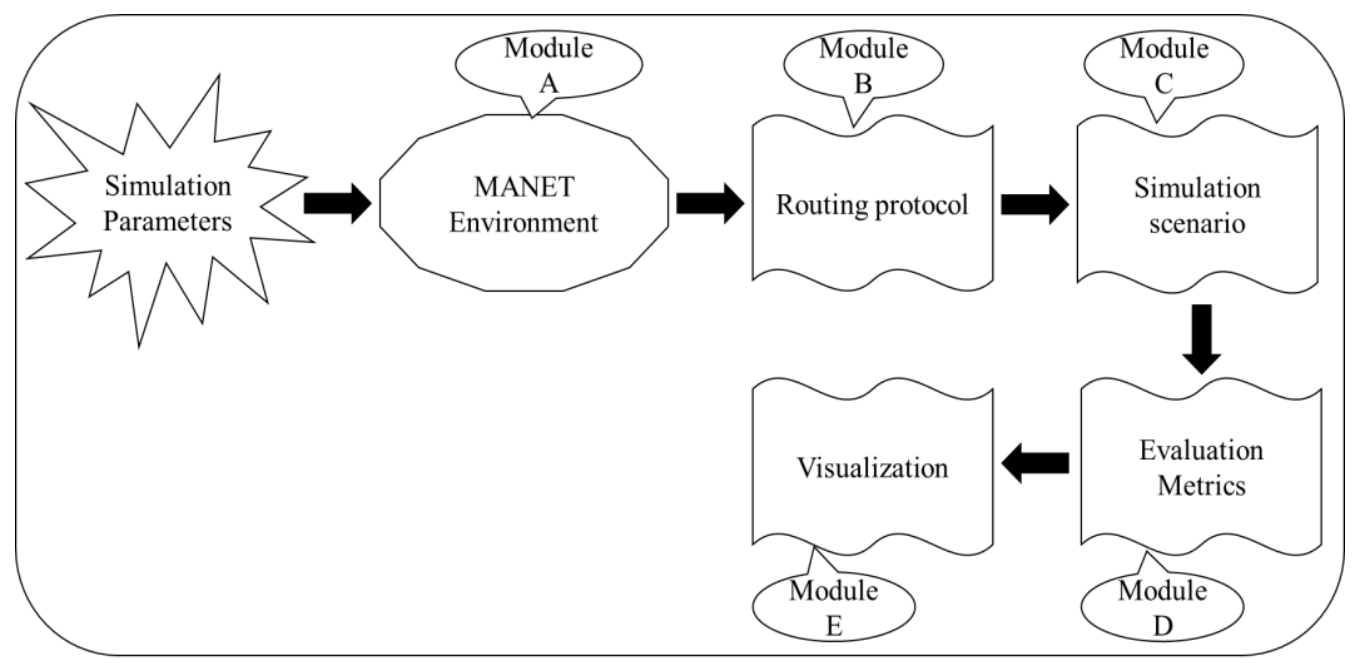

Figure 2. Simulation model design

Table 2. Simulation setting

\begin{tabular}{ccc}
\hline Parameter & Value & Unit \\
\hline Area & 1000 & $\mathrm{~m} 2$ \\
Queue size & 50 & Packet \\
Mobility Model & Random Way Point & - \\
Packet Size & 512 & Byte \\
Transmission Range & 250 & Meter \\
Protocol & AOMDV, DSDV, AODV, DSR, OLSR & - \\
No. of nodes & $(50-150: 25)$ & Node \\
Node speed & 2 & m/s \\
Simulation time & 100 & Second \\
Traffic type & CBR/UDP & - \\
\hline
\end{tabular}

This segment presents the performance evaluation simulation of the five routing conventions based on the number of hubs. Where the number of hubs changes between $(50-150: 25)$ hub. The presentation of the routing conventions is estimated depending on the TP, PDR, and E2E delay as follows.

Throughput: Throughput (TP) is referred to as the number of bits denoted by kilobits per second (Kbps) that have been effectively acknowledged or received by the destination hub [27]. TP evaluates/measures a routing convention's effectiveness in delivering packets of data to the destination. It is one of the most critical criteria in the search and rescue missions. It is measured by using (1).

$$
T P=\frac{\text { No.of bytes received } * 8}{\text { Simulation time }} * 1000 \mathrm{kbps}
$$

Packet Delivery Ratio: Packet Delivery Ratio (PDR) implies the proportion of the packets of data that got conveyed to the destination hub from some other source hubs [3, 9]. This measurement shows the routing convention's quality in its conveyance of information bundles from source to goal. The higher the Packet Delivery Ratio, the better the routing convention's performance. It is also one of the most critical criteria in the search and rescue missions. It is measured by using (2). 
$P D R=\frac{\text { No.of packet received }}{\text { No.of packets sent }} * 100 \%$

End-to-End Delay: End-to-End (E2E) delay is referred to as the meantime it takes packets of data in effectively transmitting messages over the system from source to destination hubs [11], [13]. This incorporates a wide range of delays, such as queues at interface queue; conveyance and delivery times; retransmission delays of MAC; and buffering during the latency of pathfinding. The formula expressed in Equestion 3 is used to evaluate the E2E delay:

$$
E 2 E \text { delay }=\frac{\sum_{i=1}^{n}(R i-S i)}{n}
$$

\section{RESULTS AND DISCUSSION}

This segment presents the performance evaluation simulation of the five routing conventions based on the number of hubs scenario where the number of hubs changes between 50-150 hub. The performance of the protocols is measured based on the TP, PDR, and E2E delay. It is worth mentioning, these protocols have a different performance within the variation the number of nodes. All the performance analysis results are depicted in Figures 3-5. Figure 3 illustrates the results of throughput for the AOMDV, DSDV, AODV, DSR, and OLSR routing conventions. These conventions exhibit dissimilar throughput within the variation of the number of hubs or nodes. The AODV convention betters others in execution as far as throughput is concerned. The short path separation increments reliability of the AODV and prompts limitation in loss of packets and maximum throughput. In Figure 4 are the variation of PDR for AOMDV, DSDV, AODV, DSR, and OLSR routing conventions. The varying number of hubs has a different impact on the routing protocols. At the point when there is an increment in the number, an increment in PDR occurs because of the association or link between hubs get easier. The AODV convention has better execution in terms of PDR than other conventions as a result of an establishment of a strong and short path between the source and destination hubs, which in turn reduces the loss of packets.

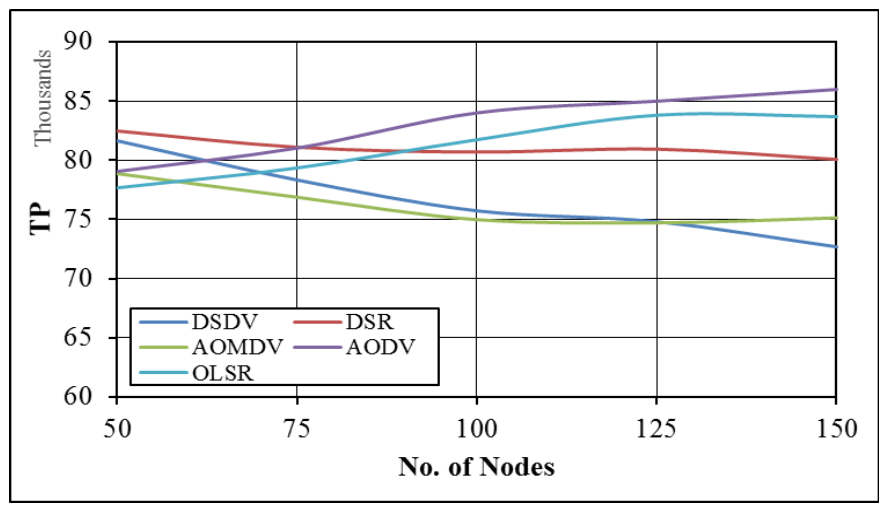

Figure 3. The TP vs number of nodes

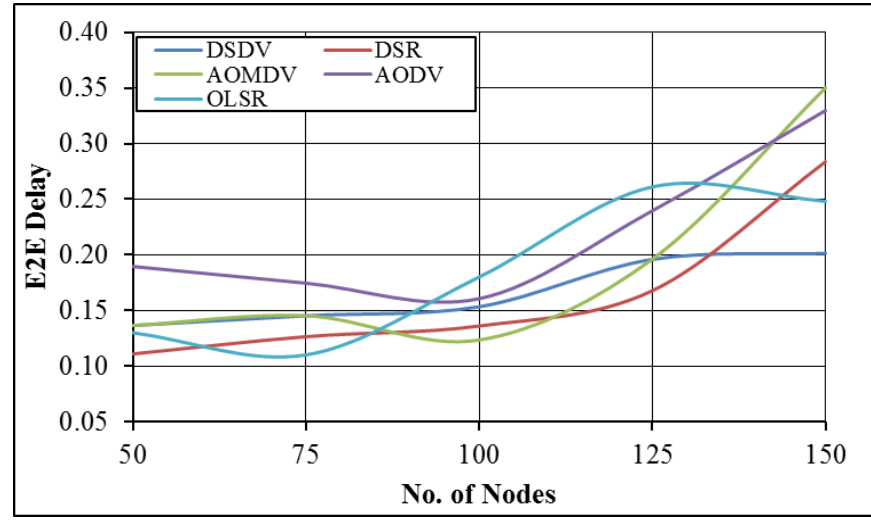

Figure 4. The PDR vs number of nodes 
Figure 5 shows the E2E delay for AOMDV, DSDV, AODV, DSR, and OLSR protocols corresponding to the number of nodes scenario. When increasing the number of nodes, the E2E delay increases as well. The OLSR routing protocol outperforms other protocols because it updates the routes information periodically, this mechanism minimizes the time taken for path selection and the packet to transfer over the network. The findings show the number of nodes scenario has a diverse influence on the behaviour of these routing protocols in this environment. However, it dramatically raises the likelihood of altering the network topology, node exhausting, increases the energy consumption, which is dramatical leads to link failure. Consequently, there is no unique protocol use in all environment. Therefore, the routing protocol selection should be done carefully due to it is depending on applications requirements.

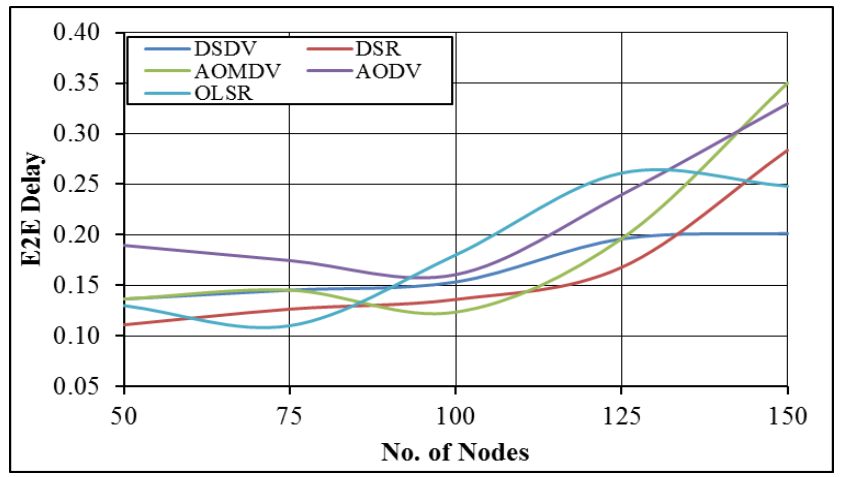

Figure 5. The E2E Delay vs number of nodes

\section{CONCLUSION}

A MANET is an assortment of remote hubs that link with one another without a framework, each node in the network utilize as a sink or a router to collect and retransmit the data packets, respectively. Various characteristics of MANET which make it a reliable and efficient network for establishing communication in a critical situation. The topology of this network changes dynamically and the hubs are allowed to move discretionarily. Several kinds of routing conventions are proposed in MANET. This paper evaluates and monitoring the performance of five routing protocols, namely; AOMDV, DSDV, AODV, DSR, and OLSR. These protocols are implemented and tested via NS2 simulator based on a number of node scenario. The outcomes demonstrate that the AODV is superior in terms of TP, and PDR. The OLSR performs better than others in terms of E2E delay.

\section{ACKNOWLEDGEMENTS}

This research was funded by the Ministry of Education Malaysia (MOE) under the Fundamental Research Grant Scheme for Research Acculturation of Early Career Researchers (FRGS-Racer) RACER/1/2019/ICT04/UTHM/1 Vote: K154.

\section{KREFERENCES}

[1] N. S.Farheen and A. Jain, "Improved Routing in MANET with Optimized Multi path routing fine tuned with Hybrid modeling," Journal of King Saud University-Computer and Information Sciences, Jan 2020.

[2] S. Zafar, et al., "Substantiation of Vulnerabilities in Various Secure Routing Protocols of MANET," In Forensic Investigations and Risk Management in Mobile and Wireless Communications, 168-194 pp. 2020.

[3] S.A. Mostafa, et al., "Social networking mobile apps framework for organizing and facilitating charitable and voluntary activities in Malaysia," Bulletin of Electrical Engineering and Informatics, vol. 9, no. 2, pp.827-833, 2020.

[4] A. Shakir, et al., "Novel Method of Improving Quality of Service for Voice over Internet Protocol Traffic in Mobile Ad Hoc Networks," International Journal of Communication Networks and Information Security, vol. 11, no. 3, pp. 331-341, Dec 2019.

[5] A. Abdali, and R.C. Muniyandi, "Optimized Model for Energy Aware Location Aided Routing Protocol in MANET," International Journal of Applied Engineering Research, vol. 12, no. 14, pp.4631-4637, 2017.

[6] M. K. Gulati and K. Kumar, "Performance comparison of mobile Ad Hoc network routing protocols," International Journal of Computer Networks \& Communications, vol. 6, 2, pp. 127-142, 2014.

[7] M. A Jubair, et al., "Bat Optimized Link State Routing Protocol for Energy-Aware Mobile Ad-Hoc Networks," Symmetry, vol. 11, no. 11, pp. 1-22, 2019. 
[8] A. Lit, et al., "Comparative performance evaluation of routing algorithm and topology size for wireless networkon-chip," Bulletin of Electrical Engineering and Informatics, vol. 8, no. 4, pp. 1239-1250, 2019.

[9] A. Sharmin, et al., "A novel bio-inspired routing algorithm based on ACO for WSNs," Bulletin of Electrical Engineering and Informatics, vol. 8, no. 2, pp. 718-726, 2019.

[10] M. Nawir, et al., "Effective and efficient network anomaly detection system using machine learning algorithm," Bulletin of Electrical Engineering and Informatics, vol. 8, no. 1, pp. 46-51, 2019.

[11] M. H. Hassan and R. C. Muniyandi, "An improved hybrid technique for energy and delay routing in mobile ad-hoc networks," International Journal of Applied Engineering Research, vol. 12, no. 1, pp. 134-139, 2017.

[12] M. H. Hassan et al., "A hybrid algorithm for improving the quality of service in MANET," International Journal on Advanced Science, Engineering and Information Technology, vol. 8, no. 4, pp. 1218-1225, 2018.

[13] M. Jubair and R. Muniyandi, "NS2 Simulator to Evaluate the Effective of Nodes Number and Simulation Time on the Reactive Routing Protocols in MANET," International Journal of Applied Engineering Research, vol. 11, no. 23, pp. 11394-11399, 2016.

[14] B. A. Khalaf, S. A. Mostafa, A. Mustapha, M. A. Mohammed and W. M. Abduallah, "Comprehensive Review of Artificial Intelligence and Statistical Approaches in Distributed Denial of Service Attack and Defense Methods," in IEEE Access, vol. 7, pp. 51691-51713, 2019.

[15] B. A. Khalaf, et al., "A Simulation Study of Syn Flood Attack in Cloud Computing Environment," REVISTA AUS pp. 188-197, 2019.

[16] S. V. R. K. Rao, et al., "Wireless sensor Network based Industrial Automation using Internet of Things (IoT)," International Journal of Advanced Trends in Computer Science and Engineering, vol. 7, no. 6, pp. 82-86, 2018.

[17] M. A Jubair et al., "Performance Evaluation of Ad-Hoc On-Demand Distance Vector and Optimized Link State Routing Protocols in Mobile Ad-Hoc Networks," International Journal on Advanced Science, Engineering and Information Technology, vol. 8, no. 4, pp. 1277-1283, 2018.

[18] A. Saini and R. Nath, "Performance Evaluation of AODV and DSR Routing Protocols in MANET Networks," In Next-Generation Networks. Springer, Singapore, pp. 313-322, 2018.

[19] M. Kumar ,et al., "Performance variation of routing protocols with mobility and scalability in MANET," In NextGeneration Networks. Springer, Singapore, pp. 9-21, 2018.

[20] M. C. K. Reddy, A. Sujana, A. Sujita and K. Rudroj, "Comparing the throughput and delay of proactive and reactive routing protocols in mobile ad-hoc networks," 2018 2nd International Conference on Inventive Systems and Control (ICISC), Coimbatore, pp. 1278-1283, 2018.

[21] A. K. Dinesh, et al., "Efficiency Evaluation of DSR and AODV On-demand protocols in Wireless MANETs," International Journal of Advanced Research in Computer Science, vol. 8, no. 5, pp. 116-120, 2017.

[22] G. Kaur et al., "Comparative Study of the performance of existing protocols of MANET with simulation and justification of an improved Routing Protocol," International Journal of Advanced Research in Electronics and Communication Engineering IJARECE, vol. 6, no. 6, pp. 603-607, 2017.

[23] Y. Mai, et al., "Performance Comparison and Evaluation of the Routing Protocols for MANETs Using NS3," Journal of Electrical Engineering, vol. 5, pp. 187-195, 2017.

[24] N. Chandra and S. Soni, "Performance Analysis of AODV, DSR and DSDV in MANETS," International Journal of Computer Applications, vol. 122, no. 9, pp. 41-44, 2015.

[25] G. Aujla and S. Kang, "Comprehensive Evaluation of AODV, DSR, GRP, OLSR and TORA Routing Protocols with varying number of nodes and traffic applications over MANETs," IOSR Journal of Computer Engineering IOSR-JCE, vol. 9, no. 3, pp. 54-61, 2013.

[26] M. H. Hassan, et al., "Integrating African Buffalo Optimization Algorithm in AODV Routing Protocol for improving the QoS of MANET," Journal of Southwest Jiaotong University, vol. 54, no. 3, pp. 1-12, 2019.

[27] S. A. Mostafa, A. Y. C. Tang, M. H. Hassan, M. A. Jubair and S. H. Khaleefah, "A Multi-Agent Ad Hoc OnDemand Distance Vector for Improving the Quality of Service in MANETs," 2018 International Symposium on Agent, Multi-Agent Systems and Robotics (ISAMSR), Putrajaya, pp. 1-7, 2018. 